\title{
El modelo de aula inversa en el área de Máquinas y Motores Térmicos: análisis y comparación de experiencias ${ }^{1}$
}

\author{
Begoña Peña ${ }^{a}$, Ignacio Zabalza ${ }^{\text {b }}$, Eva Llera ${ }^{c}$, Sergio Usón ${ }^{\text {, }}$ \\ Universidad de Zaragoza - Departamento de Ingeniería Mecánica (España) \\ abpp@unizar.es, ${ }^{b}$ izabal@unizar.es, ${ }^{\mathrm{c}}$ ellera@unizar.es, ${ }^{\mathrm{d}}$ suson@unizar.es
}

\begin{abstract}
The traditional teaching model has lost its usefulness in an increasingly digitalized and globalized world. Therefore, in recent years the role of universities in the coming decades is being seriously questioned. If they do not create added value to attending sessions, Universities will lose students and could eventually disappear.

The flipped classroom model could be the natural answer to many of the challenges that the University must face if it wants to continue maintaining its leadership position in society.

The present work collects and compares the flipped classroom experiences carried out in several subjects of the area of Machines and Thermal Motors of the University of Zaragoza during the last three academic courses. Based on the experience acquired, guidelines for proper planning of the flipped classroom application are collected, both for laboratory sessions and master classes.
\end{abstract}

Keywords: Educational Videos, Flipped Classroom, Virtual Learning Environments, Higher Education Innovation, Engineering Learning.

\section{Resumen}

El modelo de enseñanza tradicional ha perdido su utilidad en un mundo cada vez más digitalizado y globalizado. Por ello, en los últimos años se está cuestionando seriamente el papel que jugarán las universidades en las próximas décadas. Si no crean valor añadido a las clases presenciales, perderán alumnos y podrían llegar a desaparecer.

El modelo pedagógico de aula inversa podría ser la respuesta natural a muchos de los retos a los que debe enfrentarse la Universidad si quiere seguir manteniendo su posición de liderazgo en la sociedad.

\footnotetext{
1 Este trabajo se ha desarrollado en el marco de los proyectos PIIDUZ_18_102 y PRAUZ_18_012 de innovación docente (Programa de Proyectos de Innovación Docente para Grupos de Profesores del Vicerrectorado de Política Académica de la Universidad de Zaragoza).
} 
El modelo de aula inversa en el área de Máquinas y Motores Térmicos: análisis y comparación de experiencias

El presente trabajo recoge y compara las experiencias sobre aula inversa llevadas a cabo en varias asignaturas del área de Máquinas y Motores Térmicos de la Universidad de Zaragoza durante los últimos tres cursos académicos. A partir de la experiencia adquirida, se recogen pautas para planificar adecuadamente la aplicación de aula inversa, tanto en sesiones de laboratorio como en clases magistrales.

Palabras clave: Videos didácticos, Aula inversa, Entornos Virtuales de Aprendizaje, Enseñanza universitaria, Aprendizaje en Ingeniería.

\section{Introducción}

En los últimos años se está cuestionando mucho en los medios de comunicación y en foros especializados el papel que jugarán las universidades en un mundo cada vez más digitalizado y globalizado. La enseñanza presencial deja de tener sentido en su formato tradicional basado en la transmisión del conocimiento, ya que la información sobre casi cualquier tema está al alcance de cualquiera que tenga acceso a Internet y sepa cómo buscarla. Más aún, buena parte de los contenidos de las titulaciones que actualmente se imparten quedarán obsoletos durante las próximas décadas, ya que el $85 \%$ de los trabajos a los que aspirarán nuestros estudiantes no existen en la actualidad (Alba, 2017). De hecho, los empleadores ya valoran mucho más las competencias trasversales que el conocimiento específico, porque en muchos casos la formación que necesitan aplicar queda fuera de los planes de estudios o no se profundiza lo suficiente en ella, precisamente por su especificidad, y debe realizarse en el propio lugar de trabajo.

Empiezan a ser muchas las voces críticas que afirman que las universidades deberán adaptarse para responder a las necesidades de la sociedad, si no quieren desaparecer en las próximas décadas. Las Tecnologías de la Información y de la Comunicación (TIC) permiten, por ejemplo, que en algunas universidades de EEUU los alumnos reciban clases de profesores de referencia desde otra universidad, obligando al profesorado autóctono a jugar un papel diferente (Levy, 2017). El prestigioso profesor Dan Levy de la Universidad de Harvard considera necesario que el estudiante estudie en casa con ciertos materiales, por ejemplo con esas clases a distancia, para que las clases presenciales puedan dedicarse a otras actividades con valor añadido. Ahí es donde el profesor debe ser capaz de motivar, guiar y crear un espacio de aprendizaje significativo.

Y esta es, precisamente, la descripción del modelo de aula inversa (flipped classroom model, FCM), que podría ser la respuesta natural a muchos de los retos a los que debe enfrentarse la Universidad si quiere seguir manteniendo su posición de liderazgo en la sociedad. En este sentido, el Espacio Europeo de Educación Superior ha tratado de impulsar el cambio en el modelo de enseñanza-aprendizaje centrándolo en las competencias y exigiendo, por tanto, una revisión profunda de los roles que juegan profesor y estudiante en dicho proceso (Martín, 2008; Ríos, 2010). 
Este modelo persigue que el alumno asuma un papel muy activo en su aprendizaje, trabajando de forma autónoma o colaborativa con otros compañeros con la ayuda y supervisión del profesor (Figura 1). Éste actúa de guía, rellenando las lagunas durante el aprendizaje, seleccionando los recursos y actividades y evaluando el proceso y la adquisición de competencias (Peña, 2018).

Por un lado, el modelo de aula inversa permite aplicar en el aula metodologías activas, como la simulación, el debate, el aprendizaje basado en problemas, proyectos o casos, que estimulan los niveles superiores de razonamiento (Anderson, 2001) sobre los contenidos específicos, desarrollan las habilidades procedimentales de forma aplicada y desarrollan las competencias trasversales cuya importancia ya se ha mencionado.

Por otro lado, el modelo de aula inversa integra perfectamente el uso de las TIC para explotar todo el potencial de las metodologías activas (Valentín, 2013), a la vez que acerca el proceso de enseñanza-aprendizaje a la realidad de la sociedad actual.

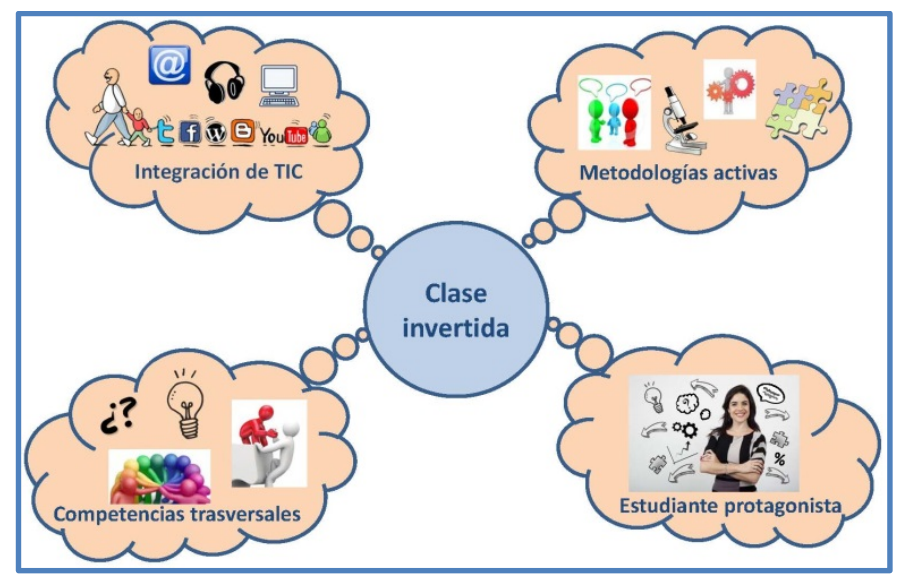

Fig. 1 Ventajas del modelo de clase inversa. Elaboración propia (Varios, 2018).

Aunque parezca que el profesor queda en segundo plano en este modelo, sigue jugando un papel crucial en el proceso de aprendizaje. Especialmente porque en la sociedad de la sobreinformación (Carr, 2019), la perspectiva del experto es esencial para guiar al alumno en el desarrollo de su razonamiento crítico que evitará la manipulación en el futuro y le permitirá gestionar eficazmente la información de forma útil.

El presente trabajo recoge y compara las experiencias sobre aula inversa llevadas a cabo en varias asignaturas del área de Máquinas y Motores Térmicos de la Universidad de Zaragoza durante los últimos tres cursos académicos. A partir de la experiencia adquirida, se recogen pautas para planificar adecuadamente la aplicación de aula inversa tanto en sesiones de laboratorio como en sesiones magistrales. 
El modelo de aula inversa en el área de Máquinas y Motores Térmicos: análisis y comparación de experiencias

\section{Objetivos}

El objetivo de esta ponencia es presentar y analizar las experiencias que han desarrollado los autores sobre FCM en los tres últimos cursos en diferentes asignaturas y titulaciones, con el fin de difundir las prácticas que han resultado positivas para que se puedan repetir en otros ámbitos. También se recogen los problemas encontrados en algunos casos para su implantación y sugerencias para mejorar su eficacia.

Los objetivos específicos de este trabajo son:

- Presentar la metodología seguida y los resultados de las diversas experiencias: observaciones, encuestas, resultados académicos.

- Comparar experiencias de la aplicación de aula inversa en sesiones de prácticas.

- Comparar experiencias de la aplicación de aula inversa en sesiones magistrales.

- Recopilar las buenas prácticas, los problemas encontrados y las estrategias propuestas para mejorar en el futuro.

\section{Desarrollo de la innovación}

\subsection{Contexto}

Las experiencias de innovación docente se han llevado a cabo durante los tres últimos cursos académicos con alumnos de diferentes titulaciones, impartidas en la Escuela de Ingeniería y Arquitectura de la Universidad de Zaragoza. En la Tabla 1 se resume la información asociada a las 5 asignaturas en las que se han realizado las experiencias en aula inversa, incluyendo: número de matriculados, número de horas impartidas bajo el modelo aula inversa, titulación, semestre y curso académico.

Tabla 1. Datos generales de las experiencias en FCM.

\begin{tabular}{|c|c|c|c|c|}
\hline Asignatura & Matriculados & $\begin{array}{l}\text { Horas } \\
\text { FCM }\end{array}$ & $\begin{array}{l}\text { Titulación } \\
\text { / Semestre }\end{array}$ & Curso \\
\hline $\begin{array}{l}\text { Termodinámica Técnica y Fund. } \\
\text { Transmisión de Calor (TT) }\end{array}$ & $\begin{array}{l}69 \\
52 \\
62\end{array}$ & 6 & GITI S3 & $\begin{array}{l}2016-2017 \\
2017-2018 \\
2018-2019\end{array}$ \\
\hline $\begin{array}{l}\text { Termodinámica Técnica y Fund. } \\
\text { Transmisión de Calor (TT) }\end{array}$ & 74 & 6 & GIQ S3 & $2017-2018$ \\
\hline Termotecnia (TC) & $\begin{array}{l}32 \\
34\end{array}$ & 3 & GIQ S6 & $\begin{array}{l}2016-2017 \\
2017-2018\end{array}$ \\
\hline $\begin{array}{l}\text { Máquinas y Motores Térmicos } \\
\text { (MMT) }\end{array}$ & $\begin{array}{c}106 \\
69\end{array}$ & 3 & GIM S6 & $\begin{array}{l}2016-2017 \\
2017-2018\end{array}$ \\
\hline $\begin{array}{l}\text { Eficiencia Energética en la } \\
\text { Edificación (EEE) }\end{array}$ & $\begin{array}{c}8 \\
10\end{array}$ & 2 & MUERyEE & $\begin{array}{l}2016-2017 \\
2017-2018\end{array}$ \\
\hline
\end{tabular}


La nomenclatura utilizada en la tabla para las diferentes titulaciones es la siguiente: GITI Grado en Ingeniería de Tecnologías Industriales, GIQ - Grado en Ingeniería Química, GIM - Grado en Ingeniería Mecánica y MUERyEE - Máster Universitario de Energías Renovables y Eficiencia Energética. Las tres primeras asignaturas son de 6 ECTS y obligatorias de grado, mientras que la última es optativa de máster de 5 ECTS.

Las asignaturas se eligieron inicialmente en base a la experiencia previa de los profesores en su impartición. El tema concreto a tratar bajo el modelo de aula inversa se ha seleccionado en base a los siguientes criterios: que tenga un carácter esencialmente aplicado, que no entrañe conceptos complejos que dificulten el aprendizaje autónomo y la disponibilidad de materiales ya desarrollados propios y ajenos.

A continuación se describe brevemente la metodología de trabajo seguida en cada asignatura.

\subsection{Termodinámica Técnica y Fundamentos de Transmisión de Calor (GITI)}

Esta asignatura de segundo curso es la base del estudio de los sistemas energéticos y, por lo tanto, esencial para la titulación. La metodología seguida se ha adaptado según el objetivo perseguido en cada caso.

Inicialmente el modelo de aula inversa tuvo como objetivo principal solventar el desfase temporal entre las prácticas y las clases de teoría. La primera se dedica al aprendizaje de un software de análisis y simulación de problemas térmicos y a afianzar el cálculo de propiedades. Las tres siguientes se centran en el análisis de ciclos termodinámicos y la última en un proceso psicrométrico, basándose dos de ellas en la toma de datos de una instalación experimental.

Con el fin de conseguir un mejor aprovechamiento de las prácticas, a través de Moodle se proporcionan con antelación una serie de materiales (guion, apuntes, videos) para que el estudiante prepare la práctica. De esta forma, el profesor apenas dedica $10 \mathrm{~min}$ a repasar los conceptos y procedimientos a aplicar y a resolver algunas dudas iniciales. Hasta el curso 2017-2018, los estudiantes debían responder a un cuestionario que entregaban en papel o enviaban a través de Moodle, mientras que en el curso 2018-2019 un cuestionario similar debe resolverse durante los primeros 10 minutos de la sesión. Si no se supera un mínimo de 4 puntos el alumno debe recuperar esa práctica en una sesión planificada a tal efecto.

En cuanto a las clases magistrales, el modelo FCM se ha aplicado en la lección completa correspondiente al cálculo de propiedades de sustancias puras y en ciertas sesiones de los temas de ciclos termodinámicos. Son temas esenciales para la asignatura y además se dispone de videos explicativos suficientes para cubrir íntegramente la materia relacionada. Estos videos se proporcionan para cada sesión junto con otros materiales a través de Moodle y las clases magistrales correspondientes se dedican a resolver dudas y problemas en pequeños grupos.

Para completar la experiencia en FCM se realizan encuestas antes y después para valorar el nivel de satisfacción de los estudiantes (Peña, 2019). 
El modelo de aula inversa en el área de Máquinas y Motores Térmicos: análisis y comparación de experiencias

\subsection{Termodinámica Técnica y Fundamentos de Transmisión de Calor (GIQ)}

En esta asignatura de segundo curso (S3), el modelo de aula inversa se ha implementado durante el curso 2017-2018 esencialmente para la preparación de las prácticas, con el fin de optimizar el aprovechamiento de las mismas, dadas las dificultades encontradas para sincronizarlas con las clases magistrales. Los temas abordados en las prácticas son: Cálculo de Propiedades Termodinámicas, Balances de Energía, Ciclos de Potencia con Turbina de Gas y Turbina de Vapor y Ciclos Frigorificos.

La metodología es similar a la seguida en el grado GITI. Con suficiente antelación, se proporciona documentación a los estudiantes para que preparen las prácticas a desarrollar en las fechas indicadas por el centro. Concretamente, se recomiendan videos y cuestionarios específicos de la materia asociada, desarrollados por los autores y proporcionados en la plataforma Moodle a través de un curso ADD en abierto (PRAUZ_17_002, 2018). Tanto la visualización de los videos, como la resolución de los cuestionarios de autoevaluación asociados a los mismos, han sido voluntarias y sin peso en la calificación de las prácticas.

Con el fin de evaluar la acogida entre los alumnos, se ha recabado información mediante encuestas acerca de la utilidad y de la calidad del material proporcionado (Zabalza, 2018).

\subsection{Termotecnia (GIQ)}

En la asignatura de Termotecnia de tercer curso (S6) se completa el estudio de los mecanismos de transferencia de calor, a partir de los conocimientos adquiridos en una asignatura previa (semestre S4). Habitualmente se detectaban problemas debido al desigual conocimiento de estos conocimientos previos por los alumnos, ya que algunos los dominaban mientras que otros los habían olvidado o arrastraban errores de concepto.

Por ello se planteó utilizar el modelo de aula inversa para repasar estos conocimientos y homogeneizar el nivel de los alumnos. En concreto, para la experiencia se ha seleccionado el tema de Conducción en Problemas Unidimensionales, al que, por ser de repaso, se le puede dedicar poco tiempo. De este modo, los alumnos adaptan el esfuerzo al grado de desconocimiento del tema que deberían dominar, lo que facilita proseguir el curso de forma satisfactoria (Peña, 2018).

Con antelación suficiente, se informa a los estudiantes de cómo y cuándo se realizará la actividad y se cuelgan en Moodle los recursos necesarios para que el estudiante repase la materia correspondiente (apuntes y ejemplos resueltos). El día de la sesión presencial, se realiza un breve repaso, se resuelven dudas y a continuación se plantean dos problemas para consolidar el aprendizaje. Al final de la sesión se ponen en común las soluciones. 


\subsection{Máquinas y Motores Térmicos (GIM)}

Esta asignatura de tercer curso (semestre S6) en el Grado de Ingeniería Mecánica, complementa a dos asignaturas básicas: Termodinámica Técnica (S2) y Transferencia de Calor (S5). El modelo de aula inversa se ha aplicado en una práctica sobre las transformaciones energéticas en una turbina de gas de varios escalonamientos de reacción que forma parte de un Ciclo de Turbina de Gas. El objetivo principal de aplicar el modelo de aula inversa es repasar los fundamentos teóricos asociados a este tema que han sido estudiados durante el primer curso de la titulación (S2), de manera que los datos de partida para el análisis de la turbomáquina (materia nueva) han de calcularse a partir del estudio del ciclo termodinámico a realizar en un tiempo anterior a la sesión de prácticas.

Es materia básica que debería ser conocida por el alumno. Sin embargo, se sabe de cursos anteriores que no la tienen bien asimilada y debe repasarse de nuevo dedicando el menor tiempo posible (Peña, 2018). Por ello, con suficiente antelación, se pone a disposición del estudiante el material necesario: guion, video específico de ciclos de turbina de gas y una plantilla de resolución. Además, con el fin de motivar la preparación de la materia asociada a la práctica, durante el curso 2017-2018 se encomendó la resolución de un cuestionario a través de Moodle antes de la sesión, que tiene un peso del $20 \%$ en la calificación de cada práctica.

Durante la sesión, la primera hora se dedica al repaso de los aspectos clave, se plantean preguntas para comprobar que se han cubierto los objetivos tras ver el video y se resuelven las dudas que hayan surgido. En las siguientes dos horas, el estudiante completa la simulación energética de la turbina con ayuda del profesor y entrega el informe de resultados al finalizar la práctica.

\subsection{Eficiencia Energética en la Edificación}

Esta asignatura optativa de Máster presenta diferencias sustanciales con respecto a las anteriores: mayor nivel académico y madurez del estudiante y un número de matriculados mucho menor.

El tema seleccionado para la experiencia de aula inversa es Arquitectura bioclimática y estrategias pasivas de ahorro energético en edificios. Para la preparación de la materia se proporciona documentación en formato texto vía Moodle y bibliografía específica. En clase se plantea un cuestionario con más de 20 preguntas para evaluar el aprendizaje sobre el tema y para abrir una discusión entre los alumnos sobre aspectos concretos, que resuelva las dudas que a priori pudieran haberse planteado con el tema. Para conseguir una amplia participación, el profesor dirige cada pregunta a un estudiante concreto. Seguidamente da el turno a los demás para poder debatir sobre la respuesta proporcionada por el estudiante. Finalmente el profesor justifica de forma razonada la respuesta correcta.

Al final de la clase, se realizan dos encuestas para conocer la opinión de los estudiantes sobre las clases universitarias y su grado de satisfacción con respecto al modelo FCM. 
El modelo de aula inversa en el área de Máquinas y Motores Térmicos: análisis y comparación de experiencias

\section{Resultados}

Los resultados aquí presentados se basan tanto en encuestas realizadas a los estudiantes como en las observaciones registradas por los profesores en plantillas desarrolladas a tal efecto (Peña, 2018). Estos formularios sirven para documentar el desarrollo de cada sesión, las impresiones generales de cada curso y las propuestas de mejora para cursos posteriores.

\subsection{Experiencias orientadas a las sesiones de prácticas}

La implementación de aula inversa para las sesiones de prácticas y laboratorio realmente no es novedosa, ya que por definición son sesiones en las que se aplican los conceptos y procedimientos explicados en las clases magistrales y estudiados previamente. Al menos en teoría, porque la realidad es que la mayoría de los estudiantes no prepara adecuadamente estas sesiones, lo que tampoco es sorprendente dado el gran volumen de trabajos a entregar y pruebas de evaluación continua en cada asignatura.

Con el fin de motivar a los estudiantes en este sentido y para solventar el frecuente problema de la desincronización temporal entre las clases magistrales y las prácticas de laboratorio, se ha planificado la aplicación del modelo de aula inversa en las asignaturas de Termodinámica Técnica y Fundamentos de Transmisión de Calor (TT-GITI y TT-GIQ) y de Máquinas y Motores Térmicos (MMT-GIM).

La metodología seguida es similar: proporcionar materiales (textos, videos) con antelación para preparar la práctica, reducir el tiempo dedicado a la explicación y dejar más tiempo para que el alumno resuelva el caso planteado. En TT-GITI se ha aplicado este modelo, junto con la resolución de un cuestionario previo (Peña, 2018), durante los últimos tres cursos académicos, mientras que para MMT-GIM se dispone de los datos los dos últimos y para TT-GIQ sólo del curso 2017-2018 (Zabalza, 2018).

Durante los primeros cursos de implementación, se comprobó a través de Moodle que sólo alrededor del $50 \%$ de los alumnos había visto el video con antelación y de hecho se detectó que algunos estudiantes estaban viendo el video durante la sesión. En la asignatura TTGITI se constató que los alumnos se copiaban el cuestionario previo o que ni siquiera lo entregaban, dado el bajo peso asignado a los mismos (10\% de la nota de prácticas). Además, pocos alumnos respondieron correctamente a las preguntas de control del profesor, indicando que dedicaron poco (o nada) de tiempo a preparar la práctica. Como resultado de esto, no se observó una mejora significativa generalizada y continuada con respecto a cursos anteriores en los que no se aplicaba aula inversa. En los próximos epígrafes se detallan los cambios introducidos durante el curso 2017-2018, así como los resultados en cada asignatura y las conclusiones generales obtenidas. 


\subsubsection{Asignatura TT-GITI}

Con el fin de motivar el trabajo previo, en la asignatura TT-GITI se ha modificado el sistema de evaluación de las prácticas durante el curso 2018-2019. En lugar de resolver fuera del aula un cuestionario sobre la materia de cada práctica, el alumno completa un test a través de Moodle durante los primeros 10 minutos de cada sesión. Cuenta 1 punto sobre la calificación de cada práctica y se debe superar un mínimo de 4 puntos, de lo contrario el alumno debe asistir a una sesión de recuperación de prácticas. Con esta medida, se han observado dos actitudes positivas en los alumnos: por un lado, abordan la práctica con un mejor conocimiento de la materia $\mathrm{y}$, por otro lado, presentan mayor motivación y concentración en el caso a resolver (Peña, 2019).

El punto de vista del estudiante es un poco diferente. Sufren cierta tensión durante la realización del cuestionario y experimentan un gran alivio una vez superado. Sin embargo, en las encuestas realizadas, tan sólo dos estudiantes indican que no consideran adecuada esta medida.

Durante el presente curso se ha contado con un profesor colaborador durante las sesiones de prácticas de TT, lo que ha permitido resolver dudas más ágilmente y hacer seguimiento detallado del proceso. Con los cambios implementados, prácticamente todos los alumnos han completado la tarea en el tiempo de la sesión con unos conocimientos claramente más sólidos.

\subsubsection{Asignatura MMT-IM}

En MMT se prepararon cuestionarios con un peso del $20 \%$ en la nota de de cada práctica para incentivar el trabajo previo. El $80 \%$ de los estudiantes respondió el cuestionario, obteniendo una nota media de 8.3. Con esta medida, se observó una mejora cualitativa en cuanto a la preparación de los estudiantes que era el objetivo inicial.

\subsubsection{Asignatura TT-GIQ}

En cuanto a TT en el grado de Ingeniería Química, la experiencia en el curso 2017-2018 fue la primera realizada en esta titulación. La acogida de los videos fue bastante sorprendente, con un número de visualizaciones totales superior a 1700, con un promedio de 115 visualizaciones por video. A pesar del carácter voluntario de los mismos, el promedio de estudiantes matriculados que accedió a los videos fue del $66 \%$, mientras que el $91 \%$ respondió a los cuestionarios de autoevaluación asociados, lo que denota que hubo algunos estudiantes que respondieron los cuestionarios sin haber visto previamente los vídeos.

En cuanto a la opinión de los estudiantes recogida mediante encuesta, el $68 \%$ consideró que visualizar los videos había contribuido a mejorar su proceso de aprendizaje y el 59\% que completar los cuestionarios de autoevaluación les había ayudado a consolidar el conocimiento presentado en los videos docentes. Con respecto a la calidad de los vídeos proporcionados, más del $88 \%$ de los estudiantes consideró que presentaban los conceptos de un modo claro y que tenían una alta calidad técnica. Asimismo a la gran mayoría les pareció que los vídeos tenían una duración adecuada y eran suficientemente amenos (Zabalza, 2018). 
El modelo de aula inversa en el área de Máquinas y Motores Térmicos: análisis y comparación de experiencias

\subsubsection{Conclusiones sobre la aplicación de aula inversa en sesiones de prácticas}

En base a las experiencias presentadas, se concluye que su aplicación conlleva una planificación sencilla para el profesor, ya que los guiones que habitualmente se elaboran incluyen el fundamento teórico que puede servir de base para desarrollar el material con el que el estudiante debe preparar la práctica. De hecho, el modelo de aula inversa en este caso no dista mucho del modelo que se ha aplicado tradicionalmente. La diferencia esencial está en la consciencia del profesor y del estudiante sobre el procedimiento, de manera que se realiza seguimiento, e incluso evaluación, del estudio previo y el enfoque durante la sesión presencial es diferente, reduciendo el tiempo dedicado a la explicación teórica.

Muchos de los videos recomendados para estas sesiones pueden coincidir con los recomendados para las sesiones magistrales y pueden ser propios o de otros autores, dada la gran cantidad de videos de calidad disponibles en Internet.

Los cuestionarios que proponemos como medida motivadora deben tener peso suficiente en la evaluación o una nota mínima para poder realizar la práctica, ya que de lo contrario los resultados pueden no ser los deseados. En ciertas disciplinas, en las que las prácticas entrañan cierto riesgo, como por ejemplo química o electricidad, que el alumno demuestre unos conocimientos mínimos es una medida imprescindible para evitar accidentes en el transcurso de las prácticas.

La implementación de los cuestionarios en Moodle es relativamente simple (siempre supeditada al tipo de preguntas disponibles que por otra parte es suficiente para este tipo de cuestionarios) y la evaluación es automática e inmediata. En cuanto al contenido, en los casos presentados se han seleccionado preguntas de dificultad media-baja, asociadas a conceptos clave o procedimientos básicos para comprender y resolver la práctica. Sin embargo, el contenido y dificultad debe adaptarse según el nivel académico y la propia disciplina.

\subsection{Experiencias orientadas a las sesiones magistrales}

En este apartado se presentan y comparan los resultados de las asignaturas de Termodinámica Técnica y Fundamentos de Transmisión de calor (grado GITI) y Termotecnia (grado GIQ). Además se extraen algunas pautas para preparar este tipo de sesiones, que consideramos que son aplicables a cualquier asignatura.

En ambos casos se ha seguido un procedimiento similar: proporcionar videos y textos para preparar la materia, resolver dudas al comienzo de la clase y dejar que los alumnos resuelvan varios ejercicios en pequeños grupos con ayuda del profesor.

Igual que en cursos anteriores se ha observado una mayor interacción alumno-profesor, lo que hace que los alumnos se sientan más motivados para resolver sus dudas y para utilizar los recursos de la asignatura. Dado el elevado número de matriculados en estas asignaturas, la presencia de un segundo profesor es muy necesaria para atender a los alumnos, ya que de lo contrario el aprovechamiento del tiempo disminuye mientras esperan turno para resolver las dudas. Durante el curso 2017-2018 se pudo contar con un profesor colaborador en TT y el rendimiento en el aula fue mayor. 
En Termotecnia del grado de Ingeniería Química no se pudo contar con el profesor adicional; sin embargo, para reducir el problema de las esperas, se comentaron el voz alta las dudas más frecuentes entre los estudiantes. Además, con la experiencia del año anterior, se adaptó el planteamiento del ejercicio al tiempo disponible: en lugar de proponer dos ejemplos desde el principio, se propone uno, dejando el segundo opcional para quienes tengan más soltura y acaben antes.

De forma general, la planificación minuciosa de estas sesiones por parte del profesor es crucial para llevar a cabo la experiencia de aula inversa con éxito. Es justo señalar que resulta casi indispensable que el profesor tenga experiencia de varios años en la impartición de la materia, ya que es necesario un conocimiento profundo, tanto de la materia en sí como de la manera en que los estudiantes la asimilan, para ser flexible y ser capaz de adaptarse de forma inmediata a los requerimientos del alumno. En este sentido, no es recomendable utilizar el modelo de aula inversa en el primer año de impartición de una asignatura.

En base a la experiencia de los autores en la aplicación de aula inversa, se resumen a continuación algunas cuestiones importantes a tener en cuenta durante la planificación de una experiencia:

- En primer lugar, el profesor deberá establecer el objetivo de aplicar el modelo de aula inversa en una asignatura y materia determinada: aprender un procedimiento, repasar materia estudiada anteriormente, aplicar conceptos difíciles de asimilar, desarrollar y evaluar competencias trasversales, etc.

- En segundo lugar, deberá decidir la metodología a aplicar en la clase presencial (aprendizaje basado en problemas, debate, técnica del puzle, etc.) y planificará las actividades a desarrollar. Es importante en este sentido prever las mayores dificultades que los alumnos suelen encontrarse en cada caso y el tiempo medio que requiere cada tarea o actividad para establecer el número de sesiones dedicado a la experiencia. De ahí la importancia de tener experiencia previa en la impartición de la materia.

- En tercer lugar, el profesor deberá decidir qué contenidos son necesarios para alcanzar el objetivo, pero que pueden trabajarse fuera del aula, y deberá seleccionar las actividades y recursos con los que el alumno preparará la sesión (cuestionarios, textos, videos, etc.). Conviene que sea materia de repaso o de comprensión sencilla, ya que si entraña excesiva dificultad o requiere una elevada inversión de tiempo puede desmotivar al estudiante y provocar el abandono.

- Con cierta antelación, es conveniente explicar al estudiante el objetivo de utilizar el modelo de aula inversa para motivarlo a preparar las sesiones y a abordar las tareas en el aula con eficacia. En este sentido el grupo de innovación IkasTHERM realiza una presentación a través de un video en su canal de youtube que puede servir de ejemplo e incluso puede ser reutilizado (IkasTHERM, 2018). 
El modelo de aula inversa en el área de Máquinas y Motores Térmicos: análisis y comparación de experiencias

- Durante la sesión, hay que estar abierto a realizar cambios y adaptarse a las sugerencias o dificultades de los alumnos del curso particular. Si por ejemplo se plantean varios problemas se debe establecer un orden de prioridad claro, guiando a los alumnos hacia los apartados más importantes, dejando para el final (por si no da tiempo) o para casa lo que es de repaso o repetitivo.

- Tras la sesión presencial, se pueden planificar tareas para afianzar o completar el aprendizaje de forma autónoma o colaborativa. Con ayuda de las TIC el seguimiento de esas actividades puede automatizarse o al menos simplificarse.

\subsection{Experiencia en asignaturas de Máster}

Tal como recoge la Tabla 1, tan sólo se ha realizado una experiencia de FCM en máster. Se trata de una asignatura optativa, con un grupo de estudiantes reducido en comparación con el resto de asignaturas de grado.

A pesar de que se observa un mayor grado de responsabilidad y motivación, se observa que algunos estudiantes responden al cuestionario basándose en el sentido común y en conocimientos previos, lo que denota que no han preparado suficientemente el tema en base al material docente proporcionado. No obstante, se aprecia un esfuerzo de los estudiantes por intentar responder correctamente las preguntas del cuestionario en clase, estando muy atentos al debate posterior a cada pregunta y a las explicaciones del profesor.

A pesar de que la documentación se proporciona con varios días de antelación a la realización del cuestionario en clase, es fundamental elegir un momento del curso adecuado, evitando tanto los periodos de mayor carga de trabajo para el estudiante como los periodos de vacaciones y festivos, para asegurar así que se estudien el material en el tiempo previsto. De otro modo, se observa que el estudio del tema queda pospuesto hasta el momento en que tengan que prepararse el examen final de la asignatura.

En cualquier caso, la aceptación de la experiencia del FCM ha sido satisfactoria en términos generales y la mayoría de los estudiantes manifiestan en las encuestas su preferencia por este modelo frente a la clase tradicional.

\section{Conclusiones}

En este trabajo se han presentado y analizado cinco experiencias sobre aula inversa en diferentes asignaturas, titulaciones y niveles académicos. En todos los casos, se concluye que es un modelo eficaz para mejorar el proceso de enseñanza-aprendizaje, ya sea en clases magistrales o de laboratorio. La acogida por parte de los alumnos ha sido muy positiva y, en su propia opinión, permite conseguir un mejor aprovechamiento de las sesiones presenciales.

Algunas de las conclusiones ya se han recogido en la sección de resultados, pero se resumen a continuación. 
- En primer lugar, consideramos que es altamente importante informar adecuadamente a los estudiantes sobre los objetivos y sobre el procedimiento de aplicación del modelo de aula inversa, para conseguir una mayor implicación y motivación.

- Independientemente del tipo de clase en el que se realice la experiencia, resulta conveniente que entre los recursos proporcionados para preparar la sesión exista algún tipo de tarea, aunque sea breve, para afianzar lo aprendido. Si se realiza telemáticamente, puede servir además para que el profesor monitorice el proceso y para que el alumno se autoevalúe.

- En grupos numerosos, la presencia de un segundo profesor es altamente recomendable siempre que sea posible, ya que de esta manera se reduce el tiempo de espera para resolver dudas y además el proceso se enriquece por la variedad de puntos de vista.

- Resulta casi imprescindible que el profesor tenga experiencia en la impartición de la materia, para prever las dificultades habituales encontradas por los alumnos y para ser capaz de adaptarse a la dinámica de la clase.

- Aplicado a las sesiones de laboratorio, este modelo conlleva una planificación sencilla para el profesor, reduce riesgos por inexperiencia en el laboratorio y permite un rendimiento mucho más alto en estas sesiones.

- Los cuestionarios a resolver antes de las sesiones, propuestos como medida motivadora, deben tener peso suficiente en la evaluación o una nota mínima en el caso de las prácticas, ya que de lo contrario puede que no se alcancen los objetivos planteados inicialmente.

- Los recursos proporcionados para las prácticas pueden coincidir con los generales de la asignatura, pero en todos los casos deben ser muy específicos del tema a tratar y con un formato lo más concentrado posible para que no desmotive al estudiante.

- En cuanto a las clases magistrales, conviene aplicarlo a materia de repaso o de comprensión sencilla, ya que si entraña excesiva dificultad o requiere una elevada inversión de tiempo puede desmotivar al estudiante y provocar el abandono.

En definitiva, consideramos que este modelo pedagógico tendrá cada vez más peso en el ámbito universitario por el marco tecnológico y social del siglo XXI y que es necesario continuar trabajando en esta dirección para fortalecer la formación del profesorado.

\section{Agradecimientos}

Los autores agradecen su colaboración en el desarrollo de materiales audiovisuales a los profesores $\mathrm{M}^{\mathrm{a}}$ Belén Zalba Nonay, José María Marín Herrero, Mariano Muñoz Rodríguez, Francisco Moreno Gómez y Luis Miguel Romeo Giménez.

\section{Referencias}

ALBA, S. (2017). Conferencia magistral en el $4^{\circ}$ Congreso Internacional de Innovación Educativa, Monterrey (Méjico) <https://www.youtube.com/watch?v=UPESF4OR-H8> [Consulta: 15 de marzo de 2019]. 
El modelo de aula inversa en el área de Máquinas y Motores Térmicos: análisis y comparación de experiencias

ANDERSON, L.W. et al. (2001). “A Taxonomy for Learning, Teaching, and Assessing: A revision of Bloom's Taxonomy of Educational Objectives”. New York: Pearson, Allyn \& Bacon.

CARR, N. (2019). “Google socava nuestra capacidad de pensar de manera profunda”. Entrevista en El País. <https://retina.elpais.com/retina/2019/03/13/tendencias/1552475304_151069.html> [Consulta: 15 de marzo de 2019].

IkasTHERM (2018). "Presentación de la metodología activa de aprendizaje". Video disponible en: $<$ https://www.youtube.com/watch?v=GUuE2yC8mjw $>$ [Consulta: 15 de marzo de 2019].

LEVY, D. (2017) "Muchas universidades van a cerrar, porque no hacen falta". Entrevista en La Vanguardia. $\quad<$ https://www.lavanguardia.com/lacontra/20170907/431099520690/muchasuniversidades-van-a-cerrar-porque-no-hacen-falta.html> [Consulta: 15 de marzo de 2019].

MARTÍN, M.M. y JUNYENT, M.V. (2008) "Learning and teaching in a context of change in the university. The promotion of teaching team”. Revista de Educación Special Issue, p. 213-234.

PEÑA, B. et al. (2018) "Experiencia piloto de aula invertida para mejorar el proceso de enseñanzaaprendizaje en la asignatura de Termodinámica Técnica”. En: Actas del congreso INRED 2018, Valencia, Editorial Universitat Politècnica de València. Doi: http:/dx.doi.org/10.4995/INRED2018.2018.8583. Disponible en: $<$ http://ocs.editorial.upv.es/index.php/INRED/INRED2018/paper/viewFile/8583/4153> [Consulta : 9 de febrero de 2019].

PEÑA, B. (2019) "Análisis del aprendizaje a través de la huella en Moodle: aplicación en la asignatura de Termodinámica Técnica" En: Actas del congreso INRED 2019, Valencia, Editorial Universitat Politècnica de València. (Enviado: 14 de marzo de 2019)

PRAUZ_17_002 (2018) "PRAUZ_17_002 - Material audiovisual de apoyo al estudio de la termodinámica y la ingeniería térmica". Curso ADD en abierto en la Universidad de Zaragoza. Disponible en: < https://moodle2.unizar.es/add/course/view.php?id=21154> [Consulta: 19 de marzo de 2019].

RÍOS, I., CAZORLA, A., DÍAZ-PUENTE, J.M. y YAGÜE, J.L. (2010) "Project-based learning in engineering higher education: two decades of teaching competences in real environments". Procedia Social and Behavioral Sciences, vol. 2, p. 1368-1378. Doi:10.1016/j.sbspro.2010.03.202.

VALENTÍN, A. et al. (2013) "Motivation and learning strategies in the use of ICTs among university students”. Computers \& Education, vol. 61, p. 52-58. Doi: 10.1016/j.compedu.2012.09.008.

VARIOS (2018). Imágenes de diferentes fuentes: http:/www.csicenlaescuela.csic.es/; http://clipground.com/image-post/59640-networking-clipart-11.jpg.html; https://www.vexels.com; commons.wikimedia.org/wiki/File:Jigsaw.svg; www.flickr.com/photos/96737006@N04/9071059572, http://www.premioscortesdecadiz.es/jovenemprendedor/roberta-medina/. [Consulta: 15 de marzo de 2019].

ZABALZA, I. et al. (2018) "Creación de un curso ADD en abierto con material audiovisual de apoyo al estudio de la termodinámica y la ingeniería térmica”. En: Libro de Actas de las XII Jornadas de Innovación Docente e Investigación Educativa UZ. Zaragoza: Universidad de Zaragoza. Disponible en: $\quad<$ https://eventos.unizar.es/22841/files/xii-jornadas-de-innovacion-docente-e-investigacioneducativa-uz.html $>$ [Consulta: 20 de marzo de 2019]. 